\title{
Über die Oxyproteinsäuren.
}

\author{
Von \\ P. Glagolew.
}

(Aus dem chemischen Laboratorium des Institutes für experimentelle Medizin.)

(Der Redaktion zugegangen am 4. Februar 1914.)

Die von Bondzynski ${ }^{1}$ ) und seinen Mitarbeitern in menschlichem und tierischem Harn gefundenen Oxyproteinsäuren scheinen einen nicht unbeträchtlichen Teil der normalen Stickstoffsubstanzen des Harnes auszumachen; nach Untersuchungen im Laboratorium von $\mathrm{Fürth}^{2}$ ) sind 3 bis $5 \%$ des Harngesamtstickstoffs auf Rechnung der Oxyproteinsäuren zu setzen.

Vom Gesichtspunkt der Stickstoffmetamorphose im allgemeinen beanspruchen diese Verbindungen, da sie größere Bruchstücke der Eiweißmolekel vorstellen, ein großes Interesse. Welche Atomgruppen werden in Form dieser komplizierten Verbindungen aus dem Organismus ausgeschieden? Sind diese Stoffe Produkte einer unvollständigen Hydrolyse, die durch Fermente, in Sonderheit durch Fermente der Gewebe und Organe weiter aufgespalten werden .können, oder stellen sie im Verhältnis zu letzteren eine Art «Antigruppe»Kühne vor?

Der Grund unserer sehr mangelhaften Kenntnisse von den Oxyproteinsäuren liegt in den Schwierigkeiten ihrer Reindarstellung und Trennung. Die einzige vorliegende Darstellungsweise, ausgearbeitet von Bondzynski ${ }^{3}$ ) und seinen Mitarbeitern, ist von Hammarsten ${ }^{4}$ ) zutreffend als «sehr mühsam und umständlich» gekennzeichnet.

1) Bullet. internat. de l'Académie de Sciences de Cracovie. Juillet 1905. Diese Zeitschrift, Bd. 46, S. 83, 1905.

$\therefore$ ) W. Ginsberg, Hofmeisters Beitr., Bd. 10, S. 411, 1907.

3) $1 . \mathrm{c}$

4) Lehrb. der physiol. Chemie, 7. Aufl., 1910, S. 714. 
Durch die Versuche von Browinski und Dombrowski ${ }^{1}$ ) ist erwiesen, daß die Oxyproteinsäuren ihrem Verhalten nach Polypeptide sind; bei Titration mit Formol nach Sörensen gelang es nämlich nachzuweisen, daß die Oxyproteinsäuren freie $\mathrm{NH}_{\mathbf{2}}$-Gruppen enthalten, und daß die Anzahl dieser Gruppen bei Hydrolyse der genannten Stoffe vermittelst Salzsäure und Fluorwasserstoffsäure bedeutend wächst. In Zusammenhang hiermit äußern die genannten Verfasser unter anderem folgende Ansicht. Nach den Untersuchungen von Henriques und Sörensen²) enthält Harn nur geringe Mengen $\mathrm{NH}_{2}$ Gruppen N, etwa 1,6-2\% des Gesamtstickstoffs. Dieselben Zahlenwerte erhielt D. van Slyke ${ }^{3}$ ) bei Benutzung seines.Verfahrens zur Bestimmung des $\mathrm{N}$ der $\left(\mathrm{NH}_{2}\right)$-Gruppen, nämlich $1,5 \%-2 ; 5 \% \mathrm{~N}$, wobei auf den *freien * Amidstickstoff bloß $0,8 \%$ des Gesamtstickstoffs entfallen. Es entsteht nun die Frage, wieweit an diesem $\mathrm{N}$ der $\mathrm{NH}_{2}$-Gruppen, der durch Formol titriert wird, die Oxyproteinsäuren beteiligt sind, und ob nicht die Gesamtmenge des $\mathrm{N}$ der $\mathrm{NH}_{2}$-Gruppen den $0 x y-$ proteinsäuren zugehört.

$\mathrm{Zu}$ dieser, auf Grund quantitativer Vergleiche der nach Sörensen titrierten $\mathrm{NH}_{\mathbf{q}}$-Gruppen, geäußerten Ansicht wäre noch hinzuzufügen, daß in Normalfällen im Harn Aminosäuren nicht mit Sicherheit nachgewiesen sind, ${ }^{4}$ ) mit alleiniger Ausnahme des Glykokolls, dessen Anwesenheit wohl durch Spaltung der sehr unbeständigen Hippursäure am einfachsten erklärt werden könnte. Andrerseits ist nicht außer acht zu lassen, daß Abderhalden und Pregl ${ }^{5}$ ) im alkoholischen Auszug von eingeengtem Harn, nach Entfernung des Harnstoffs durch Dialyse, die Anwesenheit von Polypeptiden nachgewiesen haben, die bei der Hydrolyse verschiedene Aminosäuren gaben. Zieht man nun die Löslichkeit der Oxyproteinsäuren in Alkohol, sowie ihr

1) Diese Zeitschrift, Bd. 77, S. 92, 1912.

2) Diese Zeitschrift, Bd. 60, S. 1.

3) Berichte d. deutsch. chem. Gesellsch., Bd. 43, II, S. 3179, 1910.

4) Abderhalden und Schittenhelm, Diese Zeitschrift, Bd. 47, S. 339,1906 .

5) Diese Zeitschrift, Bd. 46, S. 19, 1905. 
geringes Diffusionsvermögen in Betracht, so drängt sich unwillkürlich die Frage auf, ob es sich in den Untersuchungen von Abderhalden und Pregl nicht wenigstens zum Teil um Oxyproteinsäuren handelte.

Zwecks vorläufiger Untersuchung der Konstitution der 0xyproteinsäuren habe ich auf Anregung von Frau Dr. N. Sieber auf die aus Pferdeharn erhaltenen Baryumsalze der Oxyproteinund Alloxyproteinsäure die Methode angewandt, die von van Slyke zur Untersuchung der Spaltungsprodukte von Proteinkörpern ausgearbeitet worden ist $;^{1}$ ) hierbei wurde in den dargestellten Oxyproteinsäure-Präparaten $\operatorname{der} \mathrm{N} \operatorname{der} \mathrm{NH}_{2}$-Gruppen vor und nach der Hydrolyse bestimmt, indem die Hydrolysenprodukte durch Phosphorwolframsäure gefällt wurden; in der Fraktion, welche die durch Phosphorwolframsäure nicht fällbaren Stoffe enthielt, wurde der Gesamtstickstoff und die Gesamtziffer des $\mathrm{NH}_{2}-\mathrm{N}$ bestimmt, in der Fraktion der durch Phosphorwolframsäure fällbaren Stoffe außerdem noch Arginin und Cystin. Die Ausgangspräparate entsprachen in ihren allgemeinen Eigenschaften vollkommen den von Bondzynski²) beschriebenen und gaben auch für den Gehalt an N, Ba.und S annähernd dieselben Ziffern, die Bondzynski und seine Mitarbeiter anführen.

Das Oxyproteinbaryum ist ein weißes, außerordentlich hygroskopisches Pulver; mit Diazobenzolsulfonsäure gibt es eine sehr schwache Karminfärbuug, die auf Anwesenheit geringfügiger Mengen von Antoxyproteinsäure hinweist.

Dargestellt $6 \mathrm{~g}$.

$$
\begin{gathered}
\mathrm{N}=13,1 \% \text { nach Dumas }(0,1026 \mathrm{~g} \text { gaben bei } 17 \% \mathrm{C} . \\
\text { und } 753 \mathrm{~mm} \text { Druck } 11,8 \mathrm{ccm} \mathrm{N}) . \\
\mathrm{Ba}=29,6 \%\left(0,1130 \mathrm{~g} \text { gaben } 0,0568 \mathrm{~g} \mathrm{BaSO}_{4}\right) . \\
\mathrm{S}=0,6 \%\left(0,1240 \mathrm{~g} \text { nach Verbrennen mit } \mathrm{NaCO}_{3}+\mathrm{KNO}_{3}\right. \\
\text { gaben } \left.0,0054 \mathrm{~g} \mathrm{BaSO}_{4}\right) .
\end{gathered}
$$

Das Alloxyproteinbaryum stellt ein wenig hygroskopisches weißes Pulver vor, das nicht die geringsten Anzeichen der Diazoreaktion aufwies. und Bd. VI.

1) Abderhaldens Handbuch der Arbeitsmethoden, Bd. 5, Abt. II

2) 1. c. 
Dargestellt etwa $7 \mathrm{~g}$.

$\mathrm{N}=9,59 \%\left(0,1502 \mathrm{~g}\right.$ gaben bei $758 \mathrm{~mm}$ Druck und $\left.18^{\circ} \mathrm{C} .12,5 \mathrm{ccm} \mathrm{N}\right)$. $\mathrm{Ba}=31,0 \%\left(0,1245 \mathrm{~g}\right.$ gaben $\left.0,0656 \mathrm{~g} \mathrm{BaSO}_{4}\right)$.

$\mathrm{S}=1,89 \%\left(0,1124\right.$ gaben $0,0119 \mathrm{~g} \mathrm{BaSO}_{4}$ nach Verbrennen mit $\mathrm{Na}_{2} \mathrm{CO}_{3}+\mathrm{KNO}_{3}$ ).

Die Reindarstellung der Antoxyproteinsäure in zur Analyse genügender Menge ist uns nicht gelungen.

Hydrolyse. Als hydrolysierende Agentien gelangten $\mathrm{HCl}$ und HF zur Verwendung; hierbei wurden die Stoffe nicht eine bestimmte Anzahl Stunden der Hydrolyse ausgesetzt, wie Browinski und Dombrowski ${ }^{1}$ ) es taten ( 9 St. bei HCl bezw. 24 St. bei $\mathrm{HFl}$ ), sondern die Hydrolyse wurde so lange fortgesetzt, bis in der hydrolysierten Flüssigkeit eine Zunahme des nach van Slyke bestimmten $\mathrm{NH}_{2}$-Gruppen- $\mathrm{N}$ nicht mehr zu bemerken war. Bei Anwendung von HCl dauerte die Hydrolyse 7-9 Stunden, bei HFl etwa 40-48 Stunden; im letzteren Falle befolgten wir die Hinweise von Hugounen q und Morel, ${ }^{2}$ ) d. h. hydrolysierten mit $25 \% \mathrm{HFl}$ auf heißem Wasserbade; als Reaktionsgefäß diente ein bleierner Rundkolben mit Rückflußkühler, dessen Innenrohr aus Blei war; letzteres war mit dem Kolben vermittelst eines Stopfens aus rotem Gummi verbunden.

Die erhaltenen Resultate sind in nebenstehender Haupttabelle zusammengestellt.

Zunächst fällt ins Auge, daß bei der Hydrolyse der $\mathrm{N}$ der $\mathrm{NH}_{2}$-Gruppen anwächst, und zwar am stärksten bei Hydrolyse vermittelst Flußsäure.

\begin{tabular}{|c|c|c|c|}
\hline & \multicolumn{3}{|c|}{\begin{tabular}{l|c|c} 
Menge des $\mathrm{NH}_{2}$-Stickstoffs \\
vor der & nach der Hydrolyse \\
Hydrolyse & mit $\mathrm{HCl}$ & mit $\mathrm{HFl}$ \\
$\%$ & $\%$ & $\%$ \\
\end{tabular}} \\
\hline Alloxyproteinsaures Baryum . . . . • & $20, \overline{5}$ & 35,9 & 37,0 \\
\hline Oxyproteinsaures Baryum & 44,3 & 47,3 & 65,4 \\
\hline
\end{tabular}

1) $1 . \mathrm{c}$.

2) Bulletin de la Société chimique de France, T. 3, 1908, p: 1146. 
Haupttabelle.

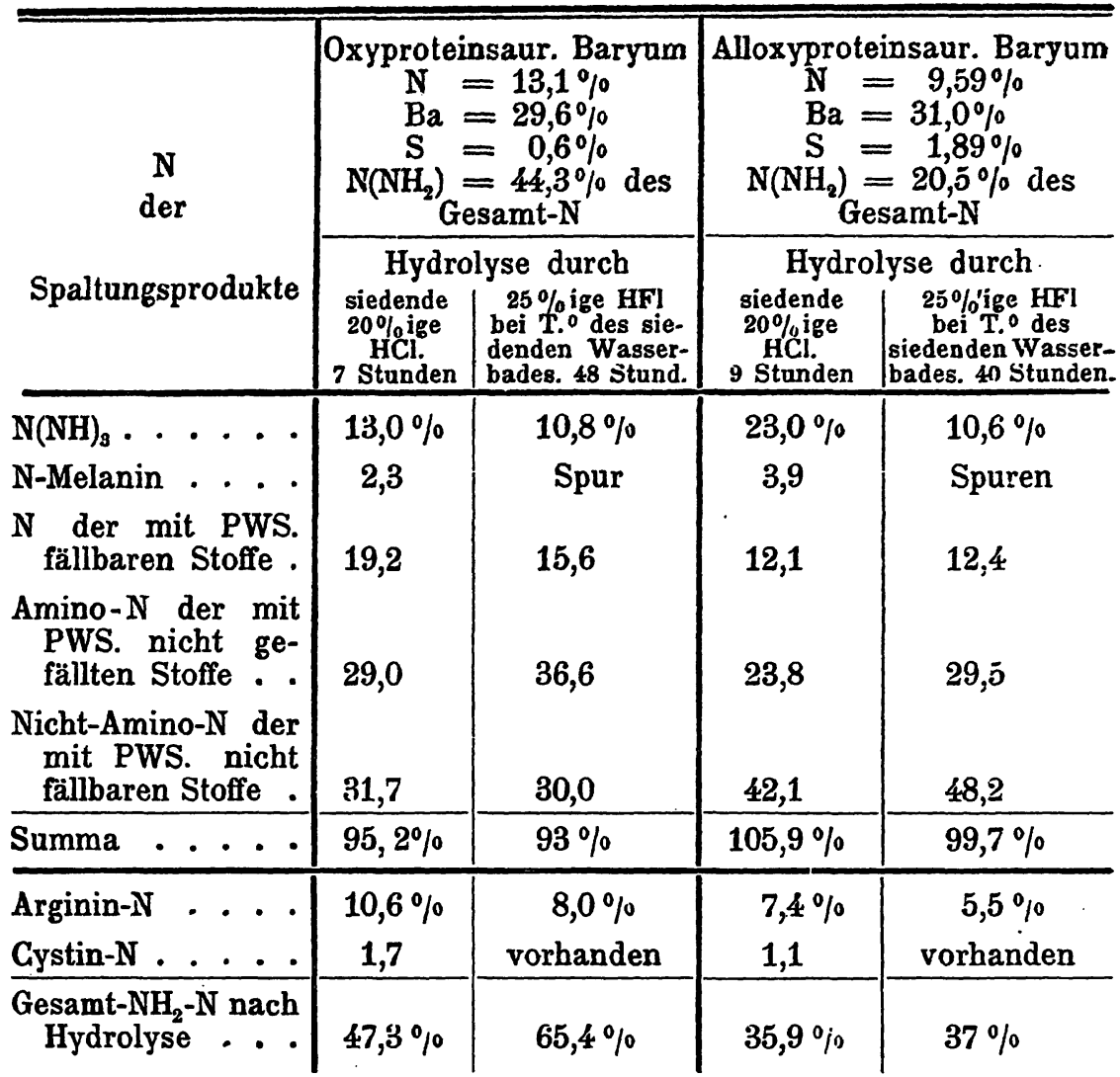

Wenden wir uns nun zur Frage, wie sich nach der Hydrolyse im Niederschlag von der Phosphorwolframsäure und im Filtrat dieses Niederschlages der $\mathrm{N}$ verteilt, so erweist sich, daß die Hauptstickstoffmenge auf die durch Phosphorwolframsäure nicht gefällten Stoffe (Aminosäuren und andere) kommt.

\begin{tabular}{|c|c|c|c|}
\hline \multirow[t]{2}{*}{ - } & \multirow{2}{*}{$\begin{array}{c}\text { Hydrolyse } \\
\text { durch }\end{array}$} & \multicolumn{2}{|c|}{$\begin{array}{c}\text { N-Gehalt der Stoffe, } \\
\text { durch Phosphorwolframsäure }\end{array}$} \\
\hline & & $\begin{array}{c}\text { nicht gefällten } \\
\%\end{array}$ & $\begin{array}{c}\text { gefällten } \\
\%\end{array}$ \\
\hline \multirow{2}{*}{ Alloxyproteinsaures Baryum } & $\mathrm{HFl}$ & 65,9 & 12,1 \\
\hline & HFl & 77,7 & 12,4 \\
\hline \multirow{2}{*}{ Oxyproteinsaures Baryum } & $\mathrm{HCl}$ & 60,7 & 19,2 \\
\hline & HEl & 66,6 & 15,6 \\
\hline
\end{tabular}


Ferner ist aus der folgenden Tabelle ersichtlich, daß für die Oxyproteinsäure und die Alloxyproteinsäure die Menge des $\mathrm{N}$ der $\mathrm{NH}_{\mathbf{2}}$-Gruppen, die in den durch Phosphorwolframsäure fällbaren und nicht fällbaren Anteilen enthalten sind, eine verschiedene ist.

\begin{tabular}{|c|c|c|c|c|c|c|c|c|}
\hline & \multirow{2}{*}{$\begin{array}{c}\text { Unter- } \\
\text { suchungs- } \\
\text { probe } \\
\text { g }\end{array}$} & \multirow{2}{*}{$\begin{array}{c}\text { Hydro- } \\
\text { lyse } \\
\text { mit }\end{array}$} & \multicolumn{3}{|c|}{$\begin{array}{l}\text { N-Menge der durch } \\
\text { PWS. nicht fällbar } \\
\text { Produkte }\end{array}$} & \multicolumn{3}{|c|}{$\begin{array}{l}\text { N-Menge der durch } \\
\text { PWS fällbaren } \\
\text { Produkte }\end{array}$} \\
\hline & & & $\begin{array}{c}\text { Ge- } \\
\text { samt- } \\
\mathbf{N} \\
\mathrm{g}\end{array}$ & $\begin{array}{l}\text { Amin } \\
\mathbf{g}\end{array}$ & $\begin{array}{r}0-\mathrm{N} \\
\%\end{array}$ & $\begin{array}{c}\text { Ge- } \\
\text { samt- } \\
\mathrm{N} \\
\mathrm{g}\end{array}$ & $\begin{array}{l}\text { Amin } \\
\mathrm{g}\end{array}$ & $\begin{array}{l}0-\mathrm{N} \\
\%\end{array}$ \\
\hline \multirow{2}{*}{$\begin{array}{l}\text { Oxyprotein- } \\
\text { saures Baryum }\end{array}$} & 1,8 & $\mathrm{HCl}$ & 0,1269 & 0,0608 & 47 & 0,042 & 0,0110 & 25 \\
\hline & 2,7 & $\mathrm{HFl}$ & 0,1870 & 0,1050 & 56 & 0,0502 & 0,0266 & 53 \\
\hline \multirow{2}{*}{$\begin{array}{l}\text { Alloxyprotein- } \\
\text { saures Baryum }\end{array}$} & 3,2 & $\mathrm{HCl}$ & $\overline{0,1555}$ & 0,0570 & $\overline{36,6}$ & $\overline{0,0301}$ & $\overline{0,0106}$ & 35 \\
\hline & 2,9 & HFl & 0,1498 & 0,0560 & 38,1 & 0,0242 & 0,0810 & 33,5 . \\
\hline
\end{tabular}

Während die maximale Amidostickstoffmenge bei der 0xyproteinsäure bis $56 \%$ des $\mathrm{N}$ der durch Phosphorwolframsäure nicht fällbaren und bis $\mathbf{5 3 \%}$ der fällbaren Stoffe beträgt, macht der Stickstoff der Aminogruppen der Alloxyproteinsäure höchstens 38.\% des Stickstoffs der nicht durch Phosphorwolframsäure fällbaren und $35 \%$ der fällbaren Stoffe aus. Somit ist die 0xyproteinsäure bedeutend reicher an Aminogruppenstickstoff.

Von den durch Phosphorwolframsäure fällbaren Stoffen beansprucht Interesse die Gegenwart von Arginin (resp. eines Stoffes, der einen Teil seines $\mathrm{N}$ in Form von Ammoniak bei längerem Kochen mit Kalihydrat abgibt) und Cystin (resp. eines Stoffes, der beimVerbrennen mit Oxydationsmitteln - Mischung von D enis - Schwefelsäure gibt). Es muß hervorgehoben werden, daß der nach van Slyke bestimmte Schwefelgehalt des Cystins im oxyproteinsauren Baryum bei Hydrolyse mit Salzsäure bis $0,4 \%$ betrug, bei einem Gesamtschwefelgehalt von $0,6 \%$, während er in der Alloxyproteinsäure bloß 0,5\% bei Hydrolyse mit Salzsäure ausmachte, bei einem Gesamtschwefelgehalt von $1,89 \%$. Es ist mir nicht gelungen, den Schwefelgehalt des in der Oxyproteinsäure nach Hydrolyse mit Fluorwasserstoff enthaltenen Cystins zu bestimmen, da nach der Verbrennung mit 
der Mischung von Denis außer dem Bariumsulfatniederschlag eine geringe Menge eines anderen Niederschlags ausfiel.

Wenden wir uns nun zum Vergleich der Ergebnisse der quantitativen Bestimmungen der Gesamtstickstoffmenge der Aminogruppen, die Browinski und Dombrowski ${ }^{1}$ ) an ihren Oxyproteinsäurepräparaten unter Anwendung der Methode von Sörensen erhalten haben, mit den von uns nach der Methode von van Slyke erzielten Resultaten. In der folgenden Tabelle entsprechen die nach Sörensen erhaltenen Zahlen den Experimentaldaten von Browinski und Dombrowski, die nach van Slyke - unseren Versuchen.

\begin{tabular}{|c|c|c|c|c|c|c|}
\hline & \multicolumn{6}{|c|}{ Amidostickstoffmenge } \\
\hline & \multicolumn{2}{|c|}{\begin{tabular}{c|} 
Vor \\
der Hydrolyse
\end{tabular}} & \multicolumn{4}{|c|}{$\begin{array}{c}\text { Nach der Hydrolyse vermittelst } \\
\mathrm{HCl}\end{array}$} \\
\hline & $\left|\begin{array}{c}\text { nach } \\
\text { Sôrensen } \\
\%\end{array}\right|$ & $\mid \begin{array}{c}\text { nach } \\
\text { v. SIyke } \\
\% \%\end{array}$ & $\left|\begin{array}{c}\text { nach } \\
\text { Sörens en } \\
0 \% 0\end{array}\right|$ & $\left|\begin{array}{l}\text { nach } \\
\text { v. Slyke } \\
\%\end{array}\right|$ & $\begin{array}{c}\text { nach } \\
\text { Sörensen } \\
0 \% 0\end{array}$ & $\mid \begin{array}{c}\text { nach } \\
\text { slyke } \\
\%\end{array}$ \\
\hline $\begin{array}{c}\text { Oxyprotein- } \\
\text { saures Baryum }\end{array}$ & 38,8 & 44,3 & 45 & 47 & 80,5 & 65,4 \\
\hline $\begin{array}{c}\text { Alloxyprotein- } \\
\text { saures Baryum }\end{array}$ & 6,3 & 20,5 & 20 & 35,9 & 76,9 & 37 \\
\hline
\end{tabular}

Aus der Tabelle geht hervor, daß für das oxyproteinsaure Baryum im allgemeinen ähnliche, aber nicht ganz gleiche Resultate, dagegen für das alloxyproteinsaure Baryum auseinandergehende Resultate erhalten wurden. Vor allem betrug der erreichte $\mathrm{NH}_{2}-\mathrm{N}$-Gehalt unseres Präparats 20,5\% des Gesamtstickstoffs, d. h. mehr als dreimal mehr, als im Präparat von Browinski. Wir neigen zur Annahme, daß dieser beträchtliche Unterschied dem Präparat zuzuschreiben ist; die Alloxyproteinsäure ist ein wenig beständiges Präparat, das bei abgeänderter Darstellungsweise abweichende Zahlenwerte ergibt. Weiter dürfte es von Interesse sein, daß die in den Hydrolysenprodukten von $\mathrm{HCl}$ und $\mathrm{HFl}$ von Dombrowski und Browinski nachgewiesenen bedeutenden Unterschiede nicht gefunden worden sind. Offenbar liegt der Grund hierzu in der abweichenden Hydrolysendauer (24 Stunden bei Browinski und

1) l. c. 
Dombrowski und 40-48 Stunden in unseren Versuchen). Auf die verschiedene Hydrolysendauer ist auch der Unterschied in den erhaltenen $\mathrm{NH}_{3}-\mathrm{N}$-Mengen zurückzuführen; von letzterem läßt sich im allgemeinen dasselbe sagen wie von den Amidostickstoffmengen; wenn in den Untersuchungen von Browinski die Ammoniakmenge nach der Hydrolyse durch Fluorwasserstoff dreimal geringer war als nach der Hydrolyse durch Salzsäure, so liegt darin wohl ein bemerkenswerter, jedoch nicht überaus ins Gewicht fallender Unterschied. Es muß aber doch darauf verwiesen werden, daß die Amidostickstoffmenge bei 7 stündiger Hydrolyse durch Salzsäure noch 1,22 mal größer ist als die Amidostickstoffmenge bei 48 stündiger Hydrolyse durch Fluorwasserstoff.

\begin{tabular}{|c|c|c|c|c|c|c|}
\hline & \multicolumn{6}{|c|}{$\mathrm{NH}_{3}-\mathrm{N}$ in $\%$} \\
\hline & \multirow{2}{*}{\multicolumn{2}{|c|}{$\begin{array}{c}\text { nach den Daten } \\
\text { von Browinski } \\
\text { u. Dombrowski, } \\
\text { hydrolysiert } \\
9 \text { Stund. } 24 \text { Stund. } \\
\text { mit HCl } \mid \text { mit HFl }\end{array}$}} & \multicolumn{4}{|c|}{$\begin{array}{c}\text { nach den Ergebnissen } \\
\text { der vorliegenden Arbeit, } \\
\text { hydrolysiert }\end{array}$} \\
\hline & & & $\begin{array}{l}\text { mit } \\
\mathrm{HCl}\end{array}$ & $\begin{array}{l}\text { Dauer } \\
\text { Stunden }\end{array}$ & $\begin{array}{l}\text { mit } \\
\text { HFl }\end{array}$ & $\begin{array}{c}\text { Dauer } \\
\text { Stunden }\end{array}$ \\
\hline Alloxyproteins. & $12,7 \%$ & $4,2 \%$ & $23 \%$ & 9 & $14 \%$ & 40 \\
\hline Oxyproteins. $\mathrm{Ba}$ & $25 \%$ & $8,3 \%$ & $13 \%$ & 7 & $10,6 \%$ & 48 \\
\hline
\end{tabular}

Somit bestätigen auch unsere Untersuchungen die Angaben von Morel und Hugounenq darüber, daß bei der Hydrolyse Fluorwasserstoff der Salzsäure vorzuziehen ist: bei Hydrolyse vermittelst Fluorwasserstoff ist die Ammoniakmenge geringer und es wird keine Melaninbildung beobachtet.

Das Dargelegte kann in folgende Sätze zusammengefaßt werden.

1. Die Oxyproteinsäuren stellen polypeptidartige Stoffe vor, da sie eine bestimmte Menge $\mathrm{NH}_{2}$-Gruppen enthalten, die durch Hydrolyse anwächst.

2. Die Oxyproteinsäure enthält eine sehr große Menge $\mathrm{NH}_{\mathrm{g}}$-Gruppen; bei Bestimmung nach van Slyke enthält sie 44,3\% des Gesamtstickstoffs an Amidostickstoff.

3. Die Hauptmenge (etwa $80 \%$ ) der bei hydrolytischer Spaltung der 0xy- und Alloxyproteinsäure entstehenden stick- 
stoffhaltigen Produkte sind durch Phosphorwolframsäure nicht fällbare Stoffe (Aminosäuren und andere).

4. Sowohl in der Oxy-als auch in der Alloxyproteinsäure gelang es, nach der Methode.von van Slyke Arginin und Cystin nachzuweisen.

5. Die Hydrolyse mit 25\% igen Fluorwàsserstoff nach Hugounenq und Morel besitzt den Vorzug, daß Melanine nicht gebildet werden und die Ammoniakmenge sogar bei 48 stündiger Hydrolyse bei der Temperatur des siedenden Wasserbades geringer ist als bei 9stündiger Hydrolyse vermittelst $20 \%$ iger siedender Salzsäure. 